\title{
EL RÉGIMEN CONSTITUCIONAL DE LOS PARTIDOS POLÍTICOS EN EL DERECHO DE LA UNIÓN EUROPEA: UN EQUILIBRIO SUI GENERIS ${ }^{1}$
}

\author{
ÁNGEL RODRÍGUEZ \\ Catedrático de Derecho Constitucional \\ Universidad de Málaga
}

\section{SUMARIO}

I. Introducción. II. Los dos elementos del régimen constitucional de los partidos políticos y su equilibrio. III. La constitucionalización del partido político europeo. IV. La constitucionalidad del partido político europeo.V. Conclusiones: el sui generis equilibrio del Estado de Partidos en la Unión Europea.

\section{INTRODUCCIÓN}

El objetivo de este trabajo es aplicar las herramientas conceptuales que usualmente se emplean para caracterizar el régimen constitucional de los partidos políticos en los Estados constitucionales a las normas que los regulan en el seno de la Unión Europea (citada en adelante como UE o «la Unión»), y ello con la intención de extraer de ese análisis algunas conclusiones sobre si ese régimen se encuentra o no en una situación equilibrada. Conviene, pues, aclarar con carácter previo dos cuestiones: en primer lugar, en qué sentido se equipara aquí la Unión Europea con un sistema «constitucional»; en segundo lugar, qué se entiende por régimen constitucional de los partidos políticos y cómo puede medirse su equilibrio.

La primera cuestión la trataremos sólo por remisión, pues no es posible detenerse ahora en los detalles del debate sobre la existencia o no de un derecho cons-

1 Una primera versión de este trabajo, con el título «Los partidos políticos desde una perspectiva supranacional europea», sirvió de base para preparar la ponencia, que permanecía inédita, presentada a las Jornadas sobre Partidos políticos y fortalecimiento de la democracia representativa celebradas en la Universidad de Valladolid los días 14 y 15 de Noviembre de 2013. Aquella ponencia ha sido ahora actualizada y reformada en su totalidad para su publicación. 
titucional europeo ni en hasta qué punto sus conclusiones avalarían la caracterización del sistema político de la Unión como un sistema constitucional, aunque con características propias ${ }^{2}$. Basta con recordar que, aunque formalmente la UE carezca de una Constitución, puede considerarse que sus Tratados tienen un carácter materialmente constitucional, y que es pacífico hablar de la existencia de un «espacio constitucional» europeo, al menos, y con ello es suficiente a los efectos que aquí interesan, en la medida en que el mismo puede justificar que el ordenamiento de la UE se estudie, como haremos a continuación en relación con las normas que regulan los partidos políticos, «en perspectiva constitucional» ${ }^{3}$.

La segunda cuestión, nuestro entendimiento del régimen constitucional de los partidos políticos y de la situación equilibrada en la que éste puede o no encontrarse, exige un mayor desarrollo. A ella dedicaremos el siguiente epígrafe, en el que se defenderá un determinado enfoque metodológico sobre la recepción en los textos constitucionales contemporáneos del denominado Estado de Partidos, enfoque que creemos aplicable, por las razones que acabamos de esbozar, a la regulación de los partidos en el seno de la Unión. Esa concepción del régimen constitucional de los partidos condicionará el desarrollo de los restantes epígrafes. Así, en el tercero, estudiaremos las normas de la UE que atribuyen a los partidos el ejercicio de determinadas funciones en su sistema político, particularmente las de carácter electoral y parlamentario. En el cuarto, analizaremos las normas que establecen mecanismos que compensan el despliegue por los partidos de esas funciones, incluyendo las que permiten aplicar instrumentos de control jurídico sobre su ejercicio. Finalmente, el epígrafe quinto se dedicará a formular algunas conclusiones sobre la relación entre las normas estudiadas en los dos epígrafes anteriores.

En 2014, la Unión Europea aprobó el Reglamento sobre el estatuto y la financiación de los partidos políticos europeos y las fundaciones políticas europeas (citado en adelante como RPPE «el Reglamento de $2014 »)^{4}$, por el cual se dota a estos partidos de «personalidad jurídica europea» y se regula su régimen jurídico. Como se ha dicho, la preocupación central del trabajo no es describir con detalle cómo se encuentran regulados los partidos políticos en el Derecho de la Unión, lo que excedería con mucho el espacio del que se dispone, sino evaluar hasta qué punto los dos elementos que es posible encontrar en esa regulación, el de atribución a los partidos de cierto protagonismo en el desenvolvimiento de la vida política de

2 Ver dos significativas contribuciones de uno de los más importantes protagonistas de ese debate en la academia, J. W., La Costituzione dell'Europa, Bologna, Il Mulino, 2003 y G. De BúrCA y J. WeILer, The Worlds of European Constitutionalism, Cambridge, Cambridge University Press, 2011.

3 Se toma la expresión de A. Carmona Contreras (editora), La Unión Europea en perspectiva constitucional, Pamplona, Aranzadi, 2008.

4 Reglamento (UE, EURATOM) 1141/2014, del Parlamento europeo y del Consejo, de 22 de octubre de 2014, sobre el estatuto y la financiación de los partidos políticos europeos y las fundaciones políticas europeas, DOUE de 4 de noviembre de 2014. Modifica el anterior Reglamento 2004/2003, de 4 de noviembre de 2003, reformado a su vez en 2007. 
la UE y el de aseguramiento de una serie de controles sobre el ejercicio de esas funciones, se encuentran o no en una situación equilibrada.

\section{LOS DOS ELEMENTOS DEL RÉGIMEN CONSTITUCIONAL DE LOS PARTIDOS POLÍTICOS Y SU EQUILIBRIO}

En agosto de 1927, Heinrich Triepel inauguraba el curso académico de la Universidad de Berlín con una conferencia que luego se haría célebre. En ella cifraba en cuatro momentos o fases las pautas que habían relacionado los partidos políticos con el Estado constitucional: oposición, ignorancia, legalización e incorporación. La primera fase se correspondería con el originario constitucionalismo liberal, cuya impronta individualista exigiría ilegalizar los cuerpos intermedios, como los partidos o los sindicatos; la segunda, con el protagonismo parlamentario, ayuno de cualquier tipo de regulación, de los partidos de notables propios de la democracia censitaria; la tercera, con la irrupción de los partidos de masas que trajo consigo el sufragio universal y la consecuente imposibilidad de seguir ignorando su existencia; y la cuarta, por último, con la plena consolidación jurídica del papel de los partidos en la dinámica de los Estados constitucionales ${ }^{5}$.

Es frecuente caer en el error de considerar que es esa última fase de «incorporación» la que se correspondería con los actuales Estados de partidos, en los que, en efecto, los partidos políticos protagonizan casi en exclusiva la vida política $^{6}$. No debe olvidarse que el Estado de partidos contemporáneo nace, desde el punto de vista constitucional, como una doble reacción: ciertamente, frente a la falta de realismo del constitucionalismo anterior, que, como recogió con agudeza Triepel, mantuvo durante mucho tiempo la ficción de que los partidos no actuaban como actores de relevancia constitucional, con importantes funciones electorales y parlamentarias, a pesar de que así era en la práctica; pero también frente a los autoritarismos propios del período entreguerras, que lograron instaurarse precisamente gracias a la acción política de un partido político que luego sería consagrado como partido único ${ }^{7}$.

5 H. Triepel, La Constitución y los Partidos Políticos, Madrid, Tecnos (edición original de 1930), 2015.

6 Habitualmente se olvida que Triepel señaló las diferentes fases de la relación entre el Estado y los partidos desde un punto de vista bistórico, cuestión distinta de las transformaciones que han sufrido los partidos como objeto de regulación jurídica. Sobre esa diferencia, Francisco BASTIDA, «La relevancia constitucional de los partidos políticos y sus diferentes significados. La falsa cuestión de la naturaleza jurídica de los partidos», en J. J. GonzÁlez Encinar (editor), Derecho de Partidos, Madrid, Espasa-Calpe, pp. 69-92, en pp. 71 y ss., 1992.

7 Como se sabe, la primera recepción plena del partido político como instrumento del Estado se produce precisamente en los Estados totalitarios basados en un solo partido, como fue el caso del partido bolchevique en la Unión Soviética, el partido fascista en Italia y el partido nazi en Alemania. Ver M. García PelaYo, «El Estado de Partido único», anexo en El Estado de Partidos, Madrid, Alianza Editorial, 1986, pp. $137-$ 160. La paradoja que puede apreciarse en este tipo de Estado consiste en que «de un lado constituye la negación del Estado de Partidos y de otro el punto máximo de la escalada de la determinación del Estado por el Partido» (p. 137). 
Desde este punto de vista, hasta la fase de «incorporación» descrita por Triepel, bien podría hablarse de un continuum en el que el papel del Estado iría de mayor a menor resistencia al protagonismo de los partidos en la vida estatal. Pero en el Estado de partidos que se implanta mediante la incorporación de los partidos políticos a los nuevos textos constitucionales de las democracias europeas restauradas tras la Segunda Guerra Mundial, se introduce una nueva idea para disciplinar la relación entre el Estado y el partido político: la de compensar el protagonismo que se concede a los partidos con el establecimiento de normas que sometan a un cierto control su actividad ${ }^{8}$.

El Estado de partidos actual es, pues, heredero de una doble preocupación: por una parte, consagrar constitucionalmente el importante papel que los partidos cumplen en las democracias modernas; por otra, establecer los mecanismos necesarios para que los propios partidos no pongan en peligro la democracia. $\mathrm{Y}$ debe entenderse como la pretensión constitucional de reconocer, pero al mismo tiempo regular, para evitar sus excesos, la relevancia de los partidos en la vida pública en un contexto de democracia pluralista. Por esa razón, las formulaciones constitucionales que recogen el Estado de partidos elevan a rango constitucional, al mismo tiempo, dos realidades diferentes aunque íntimamente relacionadas: el protagonismo de los partidos y el establecimiento de límites a ese protagonismo.

La tensión que existe entre estos dos elementos del régimen constitucional de los partidos puede caracterizarse jurídicamente desde diversos puntos de vista $^{9}$. El que, a nuestro juicio, recoge mejor la realidad de la que estamos hablando es la concepción del Estado de Partidos como una garantía institucional que se incorpora a los textos constitucionales y que, a su vez, ejerce, como es propio de las garantías institucionales, cierta vis atractiva sobre determinados derechos fundamentales, en este caso el de asociación y el de participación en los asuntos públicos, que, por su parte, presentan sus propias resistencias frente a la atracción de la garantía institucional ${ }^{10}$. En todo caso, y bien desde esa específica perspecti-

8 Las diferencias esenciales entre las normas materialmente constitucionales que regulan los partidos antes y después de la II Guerra Mundial se han puesto también de relieve por P. Biglino Campos, «Intervención del legislador y selección de candidatos por los partidos políticos: una perspectiva comparada» en Teoría y Realidad Constitucional, n. ${ }^{\circ}$ 35, 2015, pp. 203-223, en p. 207.

9 M. García Pelayo (1986), la ve como la que existe entre el propio Estado de Partidos, tomado este como una «consecuencia politológica del Estado democrático de nuestro tiempo» y el Estado de Derecho (p. 119). En un sentido similar, Enrique Álvarez Conde, El Derecho de Partidos, Madrid, Colex, 2005, contrapone la «posición constitucional» de los partidos (en el capítulo II) con su «régimen jurídico» (capítulo III).

10 Para un desarrollo de esa tesis y su aplicación al Estado de Partidos que instaura la CE, ver Á. RoDríguez, Transición Política y Consolidación Constitucional de los Partidos Políticos, Madrid, Centro de Estudios Constitucionales, 1989, especialmente pp. 201 y ss. La idea se recoge de M. García Pelayo (1986), para el que «la constitucionalización de los partidos parece significar, en principio — y con abstracción de los desarrollos jurisprudenciales y doctrinales de cada país - que una lesión a éstos es tanto como una lesión a la Constitución, de modo que, en términos generales que habrá que precisar en cada caso, parecen protegidos no sólo por las garantías propias de un derecho fundamental, sino también por la garantía institucional» (p. 51). 
va o desde otras, es pacífico encontrar cierta tensión entre los dos elementos del régimen constitucional de los partidos, protagonismo por una parte y control por otra $^{11}$. Una tensión que viene de la constatación de que «una democracia de partidos requiere de una regulación jurídica del sistema de partidos», puesto que «el buen funcionamiento de los partidos políticos no proviene de sí mismo» ${ }^{12}$.

En este trabajo, la tensión estructural entre protagonismo y control será descrita en adelante como la que se manifiesta entre dos principios presentes en la recepción constitucional del Estado de Partidos, el principio de constitucionalización y el principio de constitucionalidad. En los términos que emplearemos aquí, en todo Estado de partidos, el principio de constitucionalización debe estar suficientemente compensado por el principio de constitucionalidad. Es desde esa perspectiva que puede hablarse del equilibrio, o de la falta de éste, entre ambos elementos ${ }^{13}$.

Existirá una situación equilibrada si las normas que desarrollan el principio de constitucionalidad en un sentido amplio, es decir, los controles que el ordenamiento establece, concebidos como contrapesos de la relevancia que otorga a los partidos el principio de constitucionalización, garantizan, en la práctica, que, en un determinado sistema constitucional, el protagonismo de los partidos en la vida institucional, parlamentaria y en las elecciones, se encontrará efectivamente sometido a límites eficientes. Por el contrario, el régimen constitucional de los partidos políticos se encontrará desequilibrado cuando las normas que regulan el funcionamiento de éstos no son capaces de evitar las «distorsiones» en la vida democrática del Estado que un excesivo protagonismo de los partidos pueden llegar a producir ${ }^{14}$. Son esas distorsiones las que pueden provocar, llegado el caso, la transformación del Estado de Partidos en partidocracia ${ }^{15}$.

11 Descrita también por M. García PELAyo (1986) del siguiente modo: «puede generarse una tensión entre los partidos como actualización del derecho subjetivo de asociación y los partidos como entidades objetivamente necesarias para la vigencia del orden democrático y, por tanto, subordinadas a la funcionalidad de dicho orden, tensión que constituye una de las dificultades para la comprensión doctrinal y para la regulación jurídica de los partidos» (p. 51).

12 M. MORLOCK, «Dos cuestiones clave en la regulación jurídica de los partidos políticos: financiación y democracia interna», en Teoría y Realidad Constitucional n. ${ }^{\circ} 35$, pp. 183-201, 2015, en p. 184 . La cita literal completa es del sigiente tenor: «Por lo general, el buen funcionamiento de los partidos políticos no proviene de sí mismo. Para construir las condiciones de un sistema democrático de partidos se requiere de reglas, así como, también, para proteger el proceso político de los peligros inherentes y propios de una democracia de partidos, esto es: una democracia de partidos requiere de una regulación jurídica del sistema de partidos. Por este motivo, el Estado asume, cada vez más, la responsabilidad de garantizar el sistema de partidos».

13 En torno a la idea de equilibrio, en un sentido similar, ver F. Flores Giménez, «Los partidos políticos: intervención legal y espacio político, a la búsqueda del equilibrio», en Teoría y Realidad Constitucional n. ${ }^{\circ}$ 35, 2015, pp. 355-381.

14 Un repaso completo de las mismas en nuestro sistema constitucional puede verse en M. Á. PreSNO Linera, Los partidos políticos y las distorsiones jurídicas de la democracia, Barcelona, Ariel, 2000.

15 En definitiva, tomando como base la taxonomía aristotélica de formas políticas «rectas» y «desviadas» (Aristóteles, Política, III, 1297a7), podría hablarse de la partidocracia como degeneración del Estado de Partidos. Desde la concepción del régimen constitucional de los partidos que aquí estamos aplicando sería 
Como se sabe, son numerosos los Estados democráticos que han acometido la regulación del régimen constitucional de los partidos políticos, desde esa doble perspectiva, en sus textos constitucionales. Tomando como ejemplo la regulación de los partidos políticos en la Constitución Española (en adelante, CE), es posible distinguir en la propia disposición constitucional que consagra nuestro Estado de Partidos, el art. $6 \mathrm{CE}$, la presencia de ambos elementos ${ }^{16}$. El primero, el principio de constitucionalización, se encontraría en la primera parte del artículo, que supone la atribución a los partidos de importantes funciones en el desenvolvimiento del Estado constitucional: expresar el pluralismo político, concurrir a la formación y manifestación de la voluntad popular y ser instrumento fundamental para la participación política. La segunda parte se dedica al establecimiento de los criterios de constitucionalidad que permiten controlar el desempeño por los partidos de las funciones que se les atribuyen en la primera parte. Se recogen dos: el principio de libertad dentro del respeto a la Constitución y la Ley y el principio de estructura interna y funcionamiento democráticos ${ }^{17}$.

Como es evidente, para calificar, en los términos descritos, el régimen constitucional de los partidos políticos desde el punto de vista de la situación de equilibrio o desequilibrio en la que podría encontrarse, no es suficiente considerar sólo las normas constitucionales que los regulan directamente, sino que hay que tener en cuenta también otras disposiciones de la Constitución que establecen instituciones o mecanismos que les pueden servir de contrapeso, como, en el caso español, las que regulan los instrumentos de democracia semidirecta (el referéndum del art. $92 \mathrm{CE}$ o la iniciativa legislativa popular del art. 87.3 CE) o las que prohíben el mandato imperativo de los miembros de las Cortes Generales (art. 67.2 CE). Además, y partiendo de ellas, normas de diverso tipo regulan determinados aspectos del régimen jurídico de los partidos políticos, contribuyendo por tanto al equilibrio o desequilibrio al incidir sobre la tensión entre el principio de constitucionalización versus principio de constitucionalidad. Así, siguiendo con el caso español, el conjunto de normas que habría que considerar incluirían, en primer lugar, la propia Ley Orgánica de Partidos Políticos (LOPP), pero también las disposiciones de la Ley Orgánica del Régimen Electoral General (LOREG) y de los Reglamentos Parlamentarios (de las dos Cámaras de las Cortes Generales y las Asambleas Legislativas de las Comunidades Autónomas) que atribuyen diversos

posible definir la partidocracia como la consecuencia de una situación de desequilibrio, en la que el principio de constitucionalización de los partidos no habría sido suficientemente compensado por el principio de constitucionalidad. Ver Á. Rodríguez, «El Estado de Partidos y algunas cuestiones de Derecho Electoral», en Revista de Derecho Político n. ${ }^{\circ}$ 31, 1990, pp. 89-122.

16 Ver Á. Rodríguez, «El artículo 6 de la Constitución: los partidos políticos» en Revista de Derecho Político 36, 1992, pp. 47-64.

17 El texto del art. 6 CE es el siguiente: «Los partidos políticos expresan el pluralismo político, concurren a la formación y manifestación de la voluntad popular y son instrumento fundamental para la participación política. Su creación y el ejercicio de su actividad son libres dentro del respeto a la Constitución y a la ley. Su estructura interna y funcionamiento deberán ser democráticos». 
tipo de protagonismo a los partidos. Normas todas ellas en las que, como es también obvio, juega un papel transcendental la interpretación que de las mismas haya hecho el Tribunal Constitucional ${ }^{18}$.

Pues bien, como se dijo anteriormente, también en el ámbito de la Unión Europea puede analizarse el régimen constitucional de los partidos políticos desde esa doble perspectiva, pues también aquí existen normas que, por una parte, aseguran un papel de cierto protagonismo de los partidos en el desenvolvimiento de la vida institucional de la Unión (principio de constitucionalización) y otras que se encuentran más bien encaminadas a establecer mecanismos de control en el desempeño por los partidos de las funciones que se les atribuyen (principio de constitucionalidad). Esas normas se encuentran tanto en el derecho originario (los tratados que forman la «Constitución» de la UE) como en el derecho derivado, y su análisis conjunto permite esbozar algunas hipótesis acerca de si en la regulación de los partidos en el seno de la UE prima el protagonismo sobre el control o por el contrario ambos principios se encuentran en una situación que cabría calificar, en el sentido señalado, como próxima al equilibrio.

\section{LA CONSTITUCIONALIZACIÓN DEL PARTIDO POLÍTICO EUROPEO}

En términos generales, el protagonismo de los partidos políticos se consagra en los ordenamientos de los modernos Estados de partidos, desplegando lo que aquí hemos denominado principio de constitucionalización, mediante disposiciones constitucionales que proclaman, con carácter general, su relevancia como vía para canalizar la participación política de los ciudadanos o como instrumento para expresar el carácter plural de la sociedad civil. Estas proclamaciones genéricas suelen concretarse en normas de carácter más específico, que establecen el papel de los partidos en los procesos electorales y en el funcionamiento de los parlamentos y, en menor medida, de los gobiernos. Todas esas normas están también presentes, de un modo u otro, en el ordenamiento de la UE.

\section{Los partidos políticos europeos como instrumentos de participación}

La proclamación del papel de los partidos en la vida democrática de la Unión se hace en el propio Tratado de la Unión Europea (TUE), que establece que «los partidos políticos a escala europea contribuirán a formar la conciencia europea y

18 Que cuenta ya con un copioso cuerpo de doctrina sobre el particular, con algunos importantes puntos de inflexión, como el protagonizado por la STC 48/2003, que decidió el recurso de inconstitucionalidad contra la LOPP. Al respecto, L. DíAz BuEso, «Balance de quince años de jurisprudencia constitucional sobre partidos políticos», en Teoría y Realidad Constitucional n. ${ }^{\circ}$ 35, 2015, pp. 637-661. 
a expresar la voluntad de los ciudadanos de la Unión» ${ }^{19}$. La disposición conserva el mismo tenor literal que ya tenía en versiones anteriores del TUE y en el fallido proyecto de Tratado Constitucional. Ciertamente, puede parangonarse con disposiciones similares de las constituciones de los Estados miembros (por ejemplo, como se ha visto, la española), no sólo por su contenido, sino también por su ubicación sistemática en el correspondiente texto constitucional (el Título preliminar de la CE en el caso español, el Título II, dedicado a las disposiciones sobre los principios democráticos, en el caso del TUE).

También con rango «constitucional», el Tratado de Funcionamiento de la Unión Europea (TFUE) establece el título competencial en virtud del cual el legislador de la Unión podrá aprobar la correspondiente norma de desarrollo, habilitando al Parlamento Europeo (PE) y al Consejo para aprobar, mediante el procedimiento legislativo ordinario, el estatuto de «los partidos políticos a escala europea» ${ }^{20}$. En virtud de esa habilitación, se aprobó el Reglamento de 2014.

Ya un primer análisis, centrado en el carácter genérico de las declaraciones sobre la relevancia de los partidos políticos europeos (en adelante, PPE) que contienen estas normas, pone de manifiesto algunas similitudes y diferencias entre el modelo de constitucionalización de partidos de la UE y el que suele encontrarse en sus Estados miembros. El primer elemento común es que ambos modelos se fundamentan en una visión de la democracia representativa en la que los representantes lo son de los ciudadanos, no de los partidos. Es esa concepción, fiel a los postulados clásicos de la teoría de la representación, la que explica que el papel de los partidos sea sólo el de «contribuir», en la expresión del TUE («concurrir» en la de la Constitución Española, o «participar» en la de Alemania) a la formación de la voluntad popular.

Por la misma razón, en la misma disposición del TUE que estamos comentando, después de declarar que «El funcionamiento de la Unión se basa en la democracia representativa», se especifica que «Los ciudadanos estarán directamente representados en la Unión a través del Parlamento Europeo» ${ }^{21}$, y que son éstos (los ciudadanos) los titulares del «derecho a participar en la vida democrática de la Unión» ${ }^{22}$. Más adelante volveremos sobre la idea de representación directa en el Parlamento Europeo, pero interesa ahora resaltar que, en todo caso, también en la UE los eurodiputados son representantes de los ciudadanos, no de los partidos en cuyas listas concurren a las elecciones.

19 Art. 10.4 TUE.

20 Según el art 224 TFUE, «El Parlamento Europeo y el Consejo, con arreglo al procedimiento legislativo ordinario, establecerán mediante reglamentos el estatuto de los partidos políticos a escala europea, a los que se hace referencia en el apartado 4 del artículo 10 del Tratado de la Unión Europea, y en particular las normas relativas a su financiación».

21 Arts. 10.1 y 10.2 TUE.

22 «Todo ciudadano tiene derecho a participar en la vida democrática de la Unión (...)», Art. 10.3 TUE. 
Ello se confirma con las disposiciones de la Carta de Derechos Fundamentales de la Unión Europea (citada en adelante como CDFUE o «la Carta»), que establecen el derecho de asociación y el de participación política mediante el sufragio. Debido a la propia historia de la Unión, estos derechos se contemplan en apartados distintos de la Carta, el primero en el capítulo II, «Libertades» y el segundo en el capítulo V, «ciudadanía», y se atribuyen además a sujetos diferentes: el derecho de asociación a «toda persona» y el derecho de sufragio (reconocido de forma separada para los dos procesos electorales que reconoce la Carta, las elecciones al PE y las municipales), a «Todo ciudadano de la Unión ${ }^{23}$. Pero, en todo caso, queda claro que los sujetos de ambos derechos son las personas físicas (todas las personas, o sólo los ciudadanos de la Unión), no los partidos.

En definitiva, al igual que en los Estados, en la «relación representativa» que se establece entre representados y representantes en el derecho de la Unión, los partidos, jurídicamente al menos, no dejan de ser simples mediadores ${ }^{24}$. Esta idea de la democracia representativa deja abiertas importantes cuestiones (desde el transfugismo político a la titularidad del escaño) en la práctica política y en la dogmática constitucional de los propios Estados miembros que, mutatis mutandi, son pues trasladables al ámbito de la Unión.

Sin embargo, existen otras características del modo en el que los PPE «contribuyen» a formar la voluntad popular en la Unión que introducen una diferencia importante entre el modelo de constitucionalización del protagonismo de los partidos en la UE y en sus Estados miembros. Estas diferencias tienen que ver con dos fenómenos directamente ligados entre sí, hasta el punto de que pueden relacionarse como las dos caras de la misma moneda: por una parte, la inexistencia de un pueblo o demos europeo que dote de legitimidad democrática al ordenamiento jurídico de la UE de manera directa, sin intermediación de los Estados miembros; por otra, la relevancia que en el proceso decisional de la Unión se concede a los propios Estados. Ambos elementos conforman el conocido como «déficit democrático» de la Unión.

En efecto, solo la inexistencia de un auténtico demos europeo puede explicar que se atribuya a los PPE funciones directamente relacionadas con su creación o fortalecimiento. El propio TUE, como ya se ha resaltado, dispone de los partidos políticos «a escala europea» que contribuirán a «formar la conciencia política europea», es decir, a crear el sentimiento de una auténtica ciudadanía europea entre los pueblos de la Unión. La intención de atribuir esa función demosnógena a los partidos, en virtud de la cual se les encomendaría contribuir a la creación del hoy por hoy inexistente pueblo europeo, fue unas de las principales razones que

23 Arts. 12, 39 y 40 CDFUE.

24 Sobre el concepto de «relación representativa», ver el clásico de H. PitKin (1985), El concepto de representación, Madrid, Centro de Estudios Constitucionales. 
animó al legislador comunitario a aprobar el Reglamento de $2014^{25}$. Lo mismo puede decirse del apartado segundo de la propia CDFUE, que igualmente estipula, al reconocer el derecho de asociación, que «Los partidos políticos a escala de la Unión contribuyen a expresar la voluntad política de los ciudadanos de la Unión» ${ }^{26}$, una disposición cuyo contenido material (al menos en relación con el derecho de asociación) es más bien escaso y que se explica, sobre todo por su carácter simbólico ${ }^{27}$.

Las referencias a la «conciencia política europea» en el TUE y la CDFUE y las alusiones del Reglamento de 2014 a unos "partidos políticos europeos verdaderamente transnacionales» que deben esforzarse por «establecer un fuerte vínculo entre la sociedad civil europea y las instituciones de la Unión» ${ }^{28}$ dan buena cuenta de la importante función que se pretende atribuir a los partidos — ciertamente, de una manera acusadamente "voluntarista ${ }^{29}$ — para paliar el déficit democrático que padece la UE. Una de sus facetas es, como se sabe, el hecho de que la principal fuente de legitimidad de la Unión es la que le otorgan indirectamente los Estados. La intención manifiesta del legislador comunitario es que los PPE ayuden a construir una legitimidad alternativa, o cuando menos complementaria, a la que tiene su origen en cada uno de los Estados miembros.

Ello explica las disposiciones del Reglamento de 2014 que pretenden asegurar que los PPE tendrán realmente carácter europeo, asegurando su naturaleza transnacional. Así, se crea para los partidos una «personalidad jurídica europea ${ }^{30}$, estableciendo como requisito para obtenerla, entre otros a los que más adelante nos referiremos, que el partido tenga cierta presencia a lo largo del territorio de la Unión, estableciendo un número mínimo de sus Estados en los que debe desarrollar su actividad.

La idea del legislador comunitario es que sólo partidos políticos que transciendan las fronteras estatales podrán contribuir, en el sentido indicado, a paliar

25 Así lo destacó, en el debate sobre su aprobación, M. Giannakou, la ponente del informe en el que se basó el Parlamento para reformar el Reglamento 2004/2003. Ver G. LópeZ DE LA FueNTE (2014), «La novedosa propuesta de Reglamento sobre el estatuto y la financiación de los partidos políticos europeos y las fundaciones políticas europeas», en Revista de Derecho de la Unión Europea, n. ${ }^{\circ}$ 26, pp. 331-350, en p. 333.

26 Art. 12.2 CDFUE.

27 De disposición que «poco aporta» al derecho de asociación reconocido en la CDFUE la califica Miguel Pérez-Moneo (2009), «Los partidos políticos a escala europea (art. 12 CDFUE)», en J. GARCía RoCa y P. A. FERnÁNDEZ SÁNCHEZ (editores), Integración europea a través de derechos fundamentales: de un sistema binario a otro integrado, Madrid, CEPC, pp. 462-484, en p. 482. En su opinión, fue la creencia de que el fortalecimiento de los partidos políticos europeos podían contribuir a paliar el déficit democrático de la UE lo único que explica la inserción de una disposición sobre partidos políticos en la Carta (p. 468).

28 Considerandos cuarto y quinto.

29 La calificación procede de V. Constantinesco, «Los partidos políticos y sus fundaciones en el ámbito europeo: análisis a la luz del Reglamento (UE, EURATOM) n. ${ }^{\circ}$ 1141/2014 del Parlamento Europeo y del Consejo de 22 de octubre de 2014 sobre el estatuto y la financiación de los partidos políticos europeo", en Teoría y Realidad Constitucional, n. ${ }^{\circ} 35,2015$, pp. 341-354, en p. 344.

30 Art. 13 RPPE, en virtud del cual «Los partidos políticos europeos y las fundaciones políticas europeas gozarán de reconocimiento legal y capacidad jurídica en todos los Estados miembros». 
el déficit democrático de la Unión. Y, por ello, impone ese criterio como requisito para obtener la personalidad jurídica europea y acceder a las ventajas (sobre todo en materia de financiación) que se establecen. En este sentido, el Reglamento dispone que sólo puedan adquirir la personalidad jurídica europea las coaliciones de partidos nacionales que hayan obtenido algún tipo de representación parlamentaria en al menos una cuarta parte (es decir, siete) de los Estados miembros o hayan recibido en ellos al menos un $3 \%$ de los votos en las elecciones al $\mathrm{PE}^{31}$.

Esta peculiar atribución a los PPE de una específica función como actores que deben contribuir a paliar el déficit democrático de la Unión, para cuyo ejercicio se les exige desarrollar su actividad más allá de las fronteras de los Estados, es el principal elemento diferenciador del modelo de constitucionalización de los partidos políticos en la UE con respecto al de sus Estados miembros. Una función que ciertamente intenta que los partidos transciendan las fronteras estatales, pero que, paradójicamente, fija como criterio para asegurarla el de la presencia en un mínimo de Estados, de manera que no se deja de otorgar a éstos cierto protagonismo a la hora de otorgar a los partidos la personalidad jurídica europea.

Como veremos a continuación, esa misma paradoja está también presente en la normativa comunitaria que regulan el protagonismo de los PPE en los procesos electorales y en la vida parlamentaria.

\section{Los partidos políticos europeos en las elecciones}

Uno de los aspectos más sobresalientes de la constitucionalización de los partidos es el que consagra su papel en los procesos electorales. Las normas que regulan el régimen electoral son las que, como es sabido, favorecen de manera más directa el protagonismo de los partidos en las elecciones e influyen de manera decisiva en la conformación del sistema de partidos. La constitucionalización de los PPE en el ordenamiento de la UE también incluye algunas reglas que pretenden asegurar su presencia en el momento electoral, pero en ellas también son perceptibles las peculiaridades propias del modelo comunitario.

En primer lugar, es necesario poner de relieve que el protagonismo electoral de los PPE no se encuentra contemplado normativamente para todos los procesos electorales que tienen lugar en el seno de la Unión, sino sólo, y, como se verá, de manera muy matizada, para las elecciones al Parlamento Europeo. A día de hoy, los procesos electorales que tienen lugar en el seno de la Unión pueden clasificarse, desde el punto de vista competencial, en dos grandes grupos: aquellos que se

31 Según el art. 3.1.b) del Reglamento, «la coalición o sus miembros deben estar representados, en al menos una cuarta parte de los Estados miembros, por diputados al Parlamento Europeo, a los parlamentos nacionales o a los parlamentos o asambleas regionales, o la coalición o sus partidos miembros deben haber obtenido, en al menos una cuarta parte de los Estados miembros, un mínimo del $3 \%$ de los votos emitidos en cada uno de dichos Estados miembros en las últimas elecciones al Parlamento Europeo». 
encuentran bajo la competencia exclusiva de los Estados y aquellos en los que puede hablarse de una competencia compartida con la UE, que ha aprobado normas que les son también de aplicación. En este segundo grupo se encuentran las elecciones locales y las elecciones al PE. En el primero, todas las demás.

En las normas que regulan los procesos electorales que son de competencia exclusiva de los Estados miembros no existe ningún mecanismo para incentivar la concurrencia a los mismos de los PPE. Es cierto que, en algunos casos, los regímenes electorales establecidos por los Estados han introducido instrumentos que favorecen la «conciencia política europea», pero estos se han limitado a extender a los residentes nacionales de otro Estado miembro el derecho de sufragio en elecciones de carácter regional o nacional, más allá del derecho asociado a la ciudadanía de la $\mathrm{UE}^{32}$. Sin embargo, ningún Estado miembro ha regulado en modo alguno la concurrencia en esas elecciones de los PPE.

No sólo ello. Los mecanismos de incentivación de la concurrencia de los PPE se encuentran presentes en muy escasa medida en los dos procesos electorales cuya regulación comparten los Estados y la Unión, las elecciones locales y al PE. En el caso de las elecciones locales, la normativa comunitaria se limita a regular el ejercicio del derecho de sufragio por parte de los ciudadanos UE residentes en otro Estado miembro, sin considerar en absoluto la presencia en las mismas de partidos políticos europeos. En el caso de las elecciones al PE, las normas de la UE son tan sólo un poco más ambiciosas.

Aunque desde la aprobación del primer reglamento que reguló las elecciones del PE por sufragio universal, en 1976, está latente la idea de la existencia de un proceso electoral uniforme para toda la UE (principio que sigue en la actualidad consagrado en los tratados), los continuos fracasos en los intentos de aprobarlo lo han convertido en poco menos que un desiderátum. Las normas comunes llegan tan sólo a definir las condiciones para el ejercicio del derecho de sufragio por los ciudadanos de la UE residentes en otro Estado miembro, a establecer el número de escaños que corresponden a cada Estado y, desde la reforma de 2002, a exigirles que apliquen algún tipo de fórmula proporcional para su adjudicación ${ }^{33}$.

En realidad, las elecciones al PE siguen siendo 28 elecciones nacionales simultáneas, en las que el debate electoral gira sobre todo en torno a cuestiones domésticas y a la que concurren en exclusiva partidos nacionales. La actual regulación favorece en muy escasa medida que los PPE puedan contribuir en esa convocatoria electoral a la formación de la conciencia política europea. En la práctica, se reduce a contemplar (y ello sólo en una recomendación de la Comisión) la po-

32 Ver P. SÁnCheZ-Molina, «La extensión del Derecho de sufragio de los extranjeros: un análisis comparado entre los Estados miembros de la Unión Europea», en Revista Europea de Derechos Fundamentales, 2016, en prensa.

33 Decisión y Acto relativos a la elección de los diputados al Parlamento Europeo por sufragio universal directo (20 de septiembre de 1976), modificados por las Decisiones del Consejo de 25 de junio y 23 de septiembre de 2002). 
sibilidad de que en las papeletas de voto los partidos nacionales puedan incluir también, junto al suyo propio, el logo que identifique al partido político europeo al que pertenecen y a que los partidos europeos designen si lo desean un candidato a presidente de la Comisión ${ }^{34}$.

Medidas de otro tipo tendentes a asegurar el protagonismo de los PPE en las elecciones al PE podrían haber sido, sin embargo, posibles. No por la vía de limitar la participación en esos comicios de los partidos nacionales, sino incentivando la participación de los PPE de manera directa o indirecta.

La incentivación directa de la participación electoral de los PPE en esas elecciones podría haber venido de la mano de normas sobre aspectos clave de todos los procesos electorales, como las campañas electorales (sobre todo, el acceso a medios de comunicación públicos) o la financiación. Con respecto a lo primero, las normas que lo regulan, que son, como ya se ha dicho, las propias de cada Estado miembro, no contemplan la posibilidad de que los PPE puedan hacer sus propios actos de campaña electoral. De hecho, las campañas electorales propiamente europeas son prácticamente inexistentes. $\mathrm{Y}$ en cuanto a la financiación, el Reglamento de 2014 no sólo no ha contemplado la posibilidad de que la concedida a los partidos europeos pueda emplearse para financiar la concurrencia a las elecciones al PE de sus afiliados nacionales, sino que lo prohíbe expresamente $e^{35}$.

Tampoco puede hablarse de normas que incentiven el protagonismo de los partidos europeos de manera indirecta, por ejemplo mediante el establecimiento de fórmulas electorales que favorezcan las candidaturas europeas. En 2011, el PE intentó introducir un sistema de doble voto en las elecciones europeas, en virtud del cual los electores podrían votar, simultáneamente a la candidatura nacional, a una lista europea, presentada por un PPE, en la que se elegirían 25 eurodiputados. La introducción de esta fórmula estaba especialmente prevista para fortalecer la presencia en la concurrencia electoral al PE de los PPE, pero la reticencia de los Estados miembros impidió su puesta en marcha ${ }^{36}$.

\section{Los partidos políticos europeos en el Parlamento}

Asegurar el protagonismo de los partidos políticos en el desarrollo de la actividad parlamentaria es otra de las vías usuales de constitucionalización de los

34 Ver la Recomendación de la Comisión de 12 de marzo de 2013, con vistas a reforzar el desarrollo democrático y eficaz de las elecciones al Parlamento Europeo (2013/142/UE), DOUE L79, de 21.3.2013.

35 Aunque esa prohibición, cuyos fundamentos se remontan a la Sentencia del TJ 294/83, Parti écoligiste Le Verts versus Parlamento Europeo, de 23 de abril de 1986, no deja de plantear problemas. Ver Graciela López De LA Fuente (2014), Pluralismo político y partidos políticos europeos, Granada, Comares, p. 222.

36 Ver P. Mellado Prado, «El sistema de representación del Parlamento Europeo cuestionado: la ausencia de un procedimiento electoral uniforme», en Teoría y Realidad Constitucional, n. ${ }^{\circ} 33,2014$, pp. 219 42 , en p. 225. 
mismos, generalmente mediante la atribución por el reglamento de la Cámara de funciones relevantes a los grupos parlamentarios. Como ya se ha dicho, los postulados clásicos de la teoría de la representación suponen un primer límite a ese protagonismo, fundamentalmente mediante la prohibición del mandato imperativo. Esa prohibición está también presente en el Reglamento del Parlamento Europeo $^{37}$. A partir de ahí, sin embargo, el modelo europeo de constitucionalización del protagonismo parlamentario de los partidos difiere en notas muy esenciales del propio de los Estados miembros.

Para empezar, hay que resaltar que, como se acaba de ver, a las elecciones al PE no concurren PPE, sino partidos nacionales. Coherente con esta realidad, el Reglamento del PE no prevé que aquellos jueguen ningún papel a la hora de formar grupos parlamentarios. De nuevo, se emplea la técnica de exigir la presencia de diputados de varios Estados miembros para asegurar que el grupo parlamentario tenga carácter transnacional. En la versión actual del Reglamento del PE son necesarios al menos 25 diputados que provengan, como mínimo, de siete Estados miembros distintos ${ }^{38}$. Al igual que en el caso de los requisitos para inscribir un partido político europeo que contempla el Reglamento de 2014, el criterio de la multinacionalidad contemplado por el Reglamento del PE no deja de tener efectos paradójicos: consagra la imposibilidad de crear un grupo parlamentario si no se transcienden las fronteras nacionales, pero al fijarlas al mismo tiempo como criterio para su formación, consolida en cierto modo su presencia.

Todo ello no quiere decir que, junto con la diversidad territorial, la afinidad ideológica no juegue cierto papel en la conformación de los grupos parlamentarios en el PE. El Reglamento de Parlamento exige que «los diputados podrán organizarse en grupos de acuerdo con sus afinidades políticas» y las decisiones de su Mesa que han negado la constitución de «grupos técnicos», formados por diputados entre los que no había ninguna afinidad ideológica, han sido declaradas conformes a Derecho por el Tribunal de Justicia ${ }^{39}$. Pero el criterio de afinidad ideológica no llega hasta el punto de exigir que sólo diputados que pertenezcan al mismo PPE puedan formar un grupo.

Las peculiaridades del modelo europeo del Estado de Partidos están también presentes en el modo en el cual las normas de la UE disciplinan la relación de confianza entre Parlamento y Gobierno. En los regímenes parlamentarios de la mayoría de los Estados miembros, los mecanismos de otorgamiento y retirada de la confianza parlamentaria al gobierno están fuertemente condicionados por el papel que desempeñan los partidos políticos que apoyan desde el Parlamento al gobierno o que forman parte de éste. En el caso de la Unión, esa rela-

37 Art. 30.2 del Reglamento del PE.

38 Art. 30.1 del Reglamento del PE.

39 STJUE (Gran Sala) C-486/01, Front National versus European Parliament, de 29 de junio de 2004. 
ción es, como se sabe, más compleja, pues la Comisión Europea, el órgano que encarna el ejecutivo comunitario, debe contar con la confianza no sólo del Parlamento, que debe investirlo, sino también de los Estados miembros a través del Consejo. A partir de la reforma del Tratado de Lisboa, el Consejo deberá «tener en cuenta» los resultados de las elecciones europeas para proponer al presidente de la Comisión, como efectivamente hizo tras las de 2015, en la que el candidato propuesto fue el líder del partido europeo cuyas afiliaciones nacionales eran mayoritarias en la Cámara. Esa previsión, sin embargo, no asegura por completo el protagonismo de los PPE en la designación del gobierno de la Unión, ya que el Consejo sigue siendo en exclusiva titular de un discrecional derecho de propuesta de su presidente.

En realidad, no deja de ser paradójico que, dada la centralidad de los grupos parlamentarios en la génesis de los PPE desde un punto de vista histórico, aquellos tengan un protagonismo tan escaso en las normas jurídicas que los han establecido en el ordenamiento de la Unión. La paradoja existe también a nivel teórico, por ello las propuestas más sugerentes para la elaboración de un modelo para estudiar el papel de los partidos en el proceso representativo europeo siguen hablando de la necesidad de una triangulación que tenga en cuenta a los grupos parlamentarios del $\mathrm{PE}$, los partidos nacionales y los $\mathrm{PPE}^{40}$.

\section{LA CONSTITUCIONALIDAD DEL PARTIDO POLÍTICO EUROPEO}

Abordaremos en este epígrafe el estudio de los mecanismos de constitucionalidad de los PPE. Le damos ahora a este término el sentido amplio que se ha descrito supra, entendiéndolo como el conjunto de instituciones, reglas y mecanismos que, a modo de contrapeso, establecen límites o controles al protagonismo de los partidos que se deducen de su principio gemelo de constitucionalización, estudiado en el epígrafe anterior.

Al igual que los Estados miembros, los mecanismos de constitucionalidad de los partidos en el ordenamiento de la UE son susceptibles de ser estudiados desde diversos puntos de vista. A continuación, estudiaremos, en primer lugar, los instrumentos de participación política que se establecen al margen de los partidos; y, en segundo lugar, los elementos principales que definen las obligaciones que definen el régimen jurídico de los partidos políticos europeos y las sanciones que se les pueden aplicar en el caso de incumplimiento de las mismas.

40 Cfr. J. Chofre Sirvent, «El Parlamento Europeo y el déficit de partidos politicos: el protagonismo de los partidos políticos», en Revista de Derecho Constitucional Europeo, n. ${ }^{\circ} 11,2009$, pp. 363-386; Isabella M. Lo Presti, «Il Parlamento europeo ad un anno dalle elezioni: cosa è rimasto dell'euroscetticismo?», en Quaderni Costituzionale, 2015. 


\section{Instrumentos de participación al margen de los partidos políticos en la UE}

Aunque en algunos Estados el referéndum o la iniciativa legislativa popular pueden jugar, en ocasiones, un papel de cierta relevancia como contrapeso al protagonismo de los partidos políticos en el proceso representativo, lo cierto es que, con carácter general, estos mecanismos se encuentran subordinados en los ordenamientos de los Estados miembros a los de la democracia representativa. Así es también en el caso de la propia UE. En el ordenamiento comunitario no existe la posibilidad de un referéndum a nivel de toda la Unión y el único mecanismo de democracia semidirecta que se contempla, la Iniciativa Ciudadana Europea (ICE), se encuentra plagado de tantas cautelas que su funcionamiento se ha revelado poco menos que imposible ${ }^{41}$.

Las dificultades que rodean la figura de la ICE (que sus críticos consideran la causa principal del pobre balance que presenta en la actualidad) se encuentran tanto en las normas que la regulan como en los obstáculos de orden práctico que presenta su puesta en marcha. Como es habitual en estas figuras, se exige un número relativamente elevado (un millón) de firmas que avalen una iniciativa, que, además, y al igual que ocurre con en las previsiones del Reglamento de 2014 y del Reglamento del PE ya mencionadas, deben provenir de varios Estados miembros (un mínimo de una cuarta parte de los mismos, con un mínimo de firmas en cada uno de ellos ${ }^{42}$ ). Sin embargo, a diferencia de lo que ocurre con figuras afines de los Estados miembros, en caso de prosperar, los promotores de la ICE están facultados para presentarla, no ante el PE, que, como se sabe, no es titular de la iniciativa legislativa en la Unión, sino ante el ejecutivo comunitario, es decir la Comisión Europea. La presentación además, no compromete a ésta a iniciar ninguna acción significativa para su tramitación.

Por otra parte, y según se desprende de la experiencia de sus primeros años de funcionamiento, la ICE presenta también importantes problemas de carácter práctico, relacionados con la gestión de los programas informáticos necesarios para el proceso de recogida de firmas, con la protección de los datos asociados a las mismas, con la ausencia de ayuda financiera para la activación de esos procesos y con los plazos establecidos para que decaigan las iniciativas que no logran prosperar ${ }^{43}$.

En cualquier caso, como se ha dicho, la (escasa) relevancia de la ICE como contrapeso del papel de los partidos puede parangonarse con la que tienen en algunos Estados miembros (por ejemplo, España) instrumentos similares de demo-

41 La ICE se contempla en al art. 11.4 TUE, mientras que el art. 24.1 TFUE habilita al PE y al Consejo para adoptar su regulación.

42 Art. 7 del Reglamento (UE) 211/2011 del Parlamento Europeo y del Consejo, de 16 de febrero de 2011, sobre la iniciativa ciudadana.

43 Ver, al respecto, con propuestas concretas para su reforma, el informe del Servicio de Estudios del Parlamento Europeo, European Parlamentary Research Service, The European Citizen's Initiative: the experience of the first three years, PE 536.343 - April 2015 (revised version). 
cracia semidirecta. De nuevo aquí, en relación con la existencia de otros mecanismos de participación democrática que limiten la relevancia de los partidos, la peculiaridad más importante del modelo de la UE radica en el papel que su ordenamiento atribuye a los propios Estados.

En efecto, en el seno de la Unión, los Estados siguen reclamando para sí el erigirse, a través del protagonismo del Consejo en el entramado institucional y a pesar del progresivo fortalecimiento del papel del Parlamento, como los auténticos «amos» del derecho de la Unión. Si el protagonismo de los Estados es generalmente destacado (como ya se ha mencionado supra) como uno de los síntomas del déficit democrático de la UE, puede también analizarse, desde la perspectiva que interesa aquí, como límite al protagonismo de los PPE en las instituciones de la Unión, puesto que la presencia de éstos se da, sobre todo, con los matices que ya se han puesto de manifiesto, en el Parlamento.

Limitar el poder del Parlamento en beneficio del Consejo supone también, en ese sentido, limitar el protagonismo de los PPE en beneficio de los Estados. Raramente en el Consejo, a diferencia de lo que ocurre en el Parlamento, el cleavage territorial es sustituido por criterios más próximos a la party politics, como podría ser la formación de mayorías en su seno en función de las orientaciones ideológicas de los partidos en el poder en un un grupo de Estados miembros. Si hay una institución de la Unión donde el protagonismo absoluto es para los Estados, no para los partidos, esa es, sin duda, el Consejo ${ }^{44}$.

Desde un punto de vista funcional, el principal contrapeso del protagonismo de los partidos políticos en la UE no son, como en el modelo de los Estados miembros, los mecanismos de democracia semidirecta, sino el poder que, a través de su presencia en el Consejo, se otorga a los propios Estados.

\section{El régimen jurídico de los partidos europeos: los criterios de constitucionalidad}

En el sentido amplio que le estamos dando aquí al término, entenderemos que los criterios de constitucionalidad de los PPE son los que establece el ordenamiento de la UE como exigencias para poder adquirir y mantener la personalidad jurídica europea. En virtud de los mismos, se disponen mecanismos de control, y en su caso de sanción, para todos los partidos europeos que, en un determinado momento, dejen de cumplirlos.

Al igual que ocurre en muchos de sus Estados miembros, las obligaciones que establece el derecho de la Unión para con los PPE se concentran en tres gran-

44 Ver J. Tallberg y K. M. Johansson (2010), «Party Politics in the European Council», en B. Lindberg, A. Rasmussen y A. Warntjen (editores), The role of political parties in the European Union, London, Routledge, pp. 116-136. 
des campos: las de carácter financiero, las que se derivan de la exigencia de una organización interna democrática y las relacionadas con los principios y valores que los partidos deben respetar.

A pesar de su importancia capital para la calidad de la democracia representativa, las normas del Reglamento de 2014 que regulan la democracia interna de los partidos (su «gobernanza» en los términos que se emplean) establecen tan sólo algunos requisitos de carácter general, exigiendo que los Estatutos del PPE regulen aspectos como los derechos y deberes de los partidos asociados, las competencias y composición de sus órganos de gobierno, sus procesos internos de toma de decisiones, en particular los procedimientos de votación, y su política de transparencia ${ }^{45}$. Hay que tener en cuenta que los miembros de los PPE no son directamente los ciudadanos, sino los partidos nacionales, por lo que habría carecido de lógica que se hubieran regulado con detalle los derechos y deberes de los militantes como puede ocurrir con la legislación aplicable a los partidos en los Estados miembros.

Por otra parte, la exigencia de un control sobre las finanzas de los partidos es una consecuencia directa de la posibilidad de que éstos se financien con fondos públicos europeos. La financiación pública de los PPE, y de sus fundaciones, estaba ya prevista en el Reglamento 2004, cuyo objetivo principal era precisamente establecer mecanismos de financiación de «los partidos políticos a escala europea» con cargo al presupuesto comunitario. El Reglamento de 2014 mantiene esa misma finalidad de manera que las disposiciones sobre financiación ocupan buena parte de su articulado ${ }^{46}$. Las principales variaciones con respecto al sistema de control de la financiación con respecto al Reglamento anterior estriban en su carácter más detallado y en la sustitución de los controles de carácter preventivo por los que se centran en la justificación posterior de los gastos realizados ${ }^{47}$.

Sin duda, los mayores problemas jurídicos que plantea el régimen de constitucionalidad de los PPE provienen del tercero de los campos señalados, es decir, de las exigencias de carácter ideológico que se vierten sobre el catálogo de principios y valores que deben respetar. Aunque ya el anterior Reglamento de 2003 mencionaba la exigencia de que los «partidos políticos a escala europea» respetaran los valores europeos, la novedad del Reglamento de 2014 es que éste establece un detallado sistema de control sobre estos aspectos, que tanto por sus elementos sustantivos como procesales puede ser calificado como de «democracia militante».

45 Art. 4 RPPE.

46 Todo el capítulo IV, «disposiciones relativas a la financiación» (arts. 17-22) y buena parte del capítulo V, «control y sanciones» (arts. 23-30).

47 Sobre el sistema de control financiero de los PPE previsto en el Reglamento de 2014, ver V. ConsTANTinesco (2015). Para la regulación de este mismo aspecto en el Reglamento 2004/2033, ver M. D. Martínez Cuevas, "Comentario al reglamento (CE) n. ${ }^{\circ}$ 2004/2003 del Parlamento Europeo y del Consejo, de 4 de noviembre de 2003, relativo al Estatuto y la Financiación de los partidos políticos a escala europea», en Revista de Derecho Constitucional Europeo, n. ${ }^{\circ} 3,2005$, pp. 357 y ss. 
Desde un punto de vista sustantivo, el control ideológico de constitucionalidad implica por parte del PPE (y las fundaciones políticas asociadas a cada uno de ellos) la exigencia de respetar, «en particular en su programa y actividades», los valores en los que se basa la Unión tal como se enuncian en el art. 2 TUE, es decir, «el respeto de la dignidad humana, la libertad, la democracia, la igualdad, el Estado de Derecho y el respeto de los derechos humanos, incluidos los derechos de las personas pertenecientes a minorías». El respeto a esos valores es una exigencia para ser inscrito en el registro de partidos políticos europeos y para no ser dado de baja en el mismo. El sistema de control sobre su cumplimiento permite pues, si bien con características distintas, tanto actuaciones preventivas como represivas para los PPE que no lo cumplan.

En efecto, la inscripción registral, mediante la que se adquiere la personalidad jurídica europea, es, según el Reglamento de 2014, un momento idóneo para controlar si el partido europeo respeta los valores de la Unión, pues a la solicitud de inscripción deberá acompañarse una declaración formal normalizada en la que el PPE debe hacer constar expresamente su compromiso a observar, en particular en su programa y actividades, los valores recogidos en el art. 2 TUE. Ahora bien, al mismo tiempo el Reglamento especifica claramente que la declaración formal del PPE se considerará suficiente para entender cumplido, cara a la solicitud de inscripción, el requisito de respeto a los valores de la Unión. En consecuencia, cabe concluir que la única forma de denegar el registro del PPE por no respetar los valores europeos sería que éste se negara a realizar la declaración que se le exige o introdujera en la misma algún tipo de reserva. Por el contrario, el procedimiento que permite cancelar la inscripción del PPE por esta causa en un momento posterior permite un control mucho más amplio, con ocasión del cual puede comprobarse periódicamente si se siguen cumpliendo los requisitos que se exigieron para la inscripción registral, e instar, en su caso, su cancelación ${ }^{48}$.

Con carácter general, el Reglamento de 2014 prevé el establecimiento de una Autoridad independiente para los partidos políticos europeos y las fundaciones políticas europeas, a la que encarga las funciones de registro y control de los mismos $^{49}$. Es esa Autoridad la que decide sobre la inscripción de los PPE en el registro y puede imponerles medidas sancionatorias, desde multas a la cancelación de la inscripción. Aunque las sanciones previstas para los partidos por dejar de cumplir los criterios de constitucionalidad, que pueden llegar, como se acaba de decir, hasta la pérdida de la personalidad jurídica europea, pueden aplicarse por la inobservancia de cualquiera de ellos, el procedimiento previsto para el control por incumplimiento del deber de respetar los valores europeos es sustancialmente distinto del que se prevé para las sanciones por incumplimiento de las obligaciones ligadas al control financiero o a la gobernanza interna.

49 Art. 6 RPPE. 
En primer lugar, la cancelación de la inscripción registral por esa causa sólo puede adoptarse en caso de «incumplimiento manifiesto y grave» del deber de respeto de los valores europeos ${ }^{50}$. Además, el procedimiento sólo puede iniciarse a instancias del PE, el Consejo o la Comisión y exige el dictamen previo de un Comité de Personalidades Independientes de seis miembros, en el que cada una de estas instituciones nombra a dos de ellos. Finalmente, la eventual decisión de la Autoridad de cancelar la inscripción registral del PPE sólo podrá entrar en vigor si ni el PE ni el Consejo formulan objeciones a la misma ${ }^{51}$.

La atribución a órganos constitucionales como el Gobierno o el Parlamento de determinadas facultades en los procedimientos de control de constitucionalidad de partidos políticos no es extraña en el derecho de los Estados miembros (sin ir más lejos, en nuestra LOPP). Sin embargo, la contundencia del Reglamento de 2014 al disponer que la sanción de cancelación registral sólo podrá llevarse a cabo si ni el PE ni el Consejo plantean objeciones a la misma sugiere una capacidad de intervención de los órganos políticos en esos mecanismos de control muy próximos al ejercicio de un poder de veto, y que recuerdan al procedimiento similar previsto por el TUE para sancionar por vulneración de los valores europeos a un Estado miembro ${ }^{52}$.

\section{CONCLUSIONES: EL SUI GENERIS EQUILIBRIO DEL ESTADO DE PARTIDOS EN LA UNIÓN EUROPEA}

Todos los Estados miembros de la UE son, en tanto que Estados democráticos, Estados de Partidos. Al igual que hicieron la mayoría de ellos hace décadas, la propia Unión ha desarrollado recientemente las normas que definen su propio régimen constitucional de los partidos políticos, en relación con las cuales, como ocurre con los regímenes constitucionales nacionales, es posible plantearse si existe o no un relación equilibrada entre el principio de constitucionalización y el principio de constitucionalidad. Quizá la respuesta más adecuada a ese interrogante sea que el régimen constitucional de los partidos políticos en la UE se encuentra en una situación de equilibrio sui generis, pues está fuertemente condicionada por las peculiaridades del propio sistema constitucional de la Unión. Estas peculiaridades afectan tanto al despliegue de los elementos que regulan el protagonismo de los Partidos Políticos Europeos (principio de constitucionalización) como de los que garantizan el sometimiento de éste a límites y controles (principio de constitucionalidad).

En primer lugar, y en relación con el principio de constitucionalidad de los PPE, hay que recordar que una de las principales peculiaridades constitucionales

50 Art. 10.3 RPPE.

51 Art. 10.3 y 10.4 RPPE.

52 Art. 7 TUE. 
de la UE es su naturaleza de sistema multinivel, en el que las normas constitucionales de la Unión interactúan, mediante un complejo sistema de reenvíos recíprocos que no son siempre fáciles de descifrar, con las normas constitucionales de los Estados miembros. Conviene resaltar que a pesar del cambio en el nomen iuris que parece sugerir un estatus diferente de los «Partidos Políticos Europeos», tal como los denomina el Reglamento de 2014, con respecto a los «Partidos Políticos a Escala Europea», que es el nombre que sigue dándoles el TUE, el TFUE y la CDFUE y que empleaba el Reglamento de 2003, lo cierto es que aquellos siguen siendo, en la propia definición del Reglamento de 2014, una mera coalición de partidos nacionales que, en virtud de su inscripción en el registro, adquieren una personalidad jurídica europea independiente de los partidos que la forman, que siguen por lo tanto sujetos a sus propias normativas nacionales ${ }^{53}$.

El Derecho de la Unión reconoce esta situación, especificando en algunos casos qué consecuencias deben deducirse de la aplicación simultánea de las reglas nacionales y europeas a los PPE. Por ejemplo, cuando considera derecho supletorio del mismo las normas nacionales que regulan la actividad de los partidos políticos donde el PPE tenga su sede o establece que la actividad de un PPE en otro Estado miembro se regirá por lo dispuesto por la normativa nacional ${ }^{54}$. En otros casos se emplea la técnica del mínimo estándar, característica de las situaciones mutilevel, como cuando se especifica que las normas de gobernanza interna de los PPE cumplirán, además de con lo dispuesto en el Reglamento de 2014, con lo establecido para los partidos políticos nacionales por el Estado miembro en el que tenga su sede, que podrá imponerles requisitos adicionales ${ }^{55}$.

La complejidad de la situación multinivel se aprecia claramente en los problemas que presentan los mecanismos de control de constitucionalidad de los PPE relacionados con el respeto de los valores de la Unión, y que hemos calificado supra de muy próximos a los controles propios de los Estados con democracia militante. Como se sabe, este tipo de control sobre los partidos políticos existe en algunos Estados miembros, mientras que otros, por el contrario, han intentado, no sin dificultades, diseñar mecanismos de control partiendo de otros presupuestos. El control ideológico de los partidos está además, permitido por la Convención Europea de Derechos Humanos, que por una parte actúa como estándar mínimo común en todos los Estados miembros y es por otra la norma de remisión que señala la propia CDFUE ${ }^{56}$.

53 Art.2 RPPE.

54 Art. 14 RPPE.

55 Art. 4 RPPE.

56 Sobre la relación entre el sistema de control de constitucionalidad de la LOPP y el de la CEDH, ver Á. Rodríguez, «Batasuna ante el Tribunal Europeo de Derechos Humanos: protección «multinivel» de derechos en Europa y régimen de los partidos políticos en España», en Revista de Derecho Comunitario Europeo n. ${ }^{\circ}$ 35, 2010, pp. 195-221. Una síntesis de la jurisprudencia del TEDH al respecto en O. AkBULuT, «Criteria Developed by the European Court of Human Rights on the Dissolution of Political Parties», en Fordham International Law Journal n. ${ }^{\circ}$ 34, 2010, pp. 46-77. 
En este escenario, es posible que la aplicación de los instrumentos de control previstos para los PPE puedan presentar puntos de conflicto con estándares más protectores del derecho de asociación política en algunos Estados miembros. En ese caso, podría producirse tanto una convergencia entre ambos (en el que no se puede descartar un fenómeno de backsliding o regresión del estándar interno ${ }^{57}$ ) como una convivencia como órdenes separados, en donde se pueda dar, por ejemplo un PPE disuelto cuyos partidos afiliados nacionales podrían seguir funcionando por aplicación de las reglas de su derecho nacional. El propio Reglamento de 2014 parece prever esta última posibilidad, al establecer que el incumplimiento por parte de un PPE de las obligaciones derivadas del derecho nacional donde tenga su sede o desarrolle sus actividades en relación con el respeto de los valores europeos sólo podrá dar lugar por parte del Estado miembro concernido a una solicitud de que se inicie el procedimiento de cancelación registral, que sin embargo sólo podría concluir de ese modo si se cumpliesen las condiciones ya mencionadas (no planteamiento de objeciones por parte del PE o el Consejo) ${ }^{58}$.

Por último, en relación ahora con el principio de constitucionalización, la principal peculiaridad del régimen constitucional de los PPE viene dada por el hecho de que uno de los objetivos de la constitucionalización de los partidos europeos sea pretender que estos contribuyan a paliar el déficit democrático de la UE. Sin embargo, los partidos europeos tienen una limitada relevancia en el proceso de toma de decisiones de la Unión, debido al protagonismo que se concede a los propios Estados miembros o a sus partidos nacionales.

El equilibrio en el régimen constitucional de los partidos políticos no sólo evita las distorsiones de la democracia, sino que tiene también importantes efectos al fortalecer el propio sistema: a mayor institucionalización del sistema de partidos, estos actúan de una manera más predecible y dotan de una mayor estabilidad al sistema político en el que se integran ${ }^{59}$. A medio o largo plazo, una profundización en el régimen constitucional de los PPE contribuiría, muy probablemente, a fortalecer institucionalmente la Unión.

Ciertamente, el escaso tiempo transcurrido desde la entrada en vigor del Reglamento de 2014 pone de manifiesto que estamos en una situación de transición, pero hoy por hoy puede decirse que es la fuerte presencia del Estado de Partidos nacional en cada uno de sus Estados miembros uno de los principales obstáculos para la consolidación en la UE de un hipotético Estado de Partidos europeo.

57 Sobre el concepto de backsliding, ver, A. T. GuZman y Katerina Linos (2014), «Human Rights Backsliding», en California Law Review, n. ${ }^{\circ} 102$, pp. 603-654.

58 Art. 16.3 RPPE.

59 En este sentido, C. SKaCH (2012), «Political Parties and the Constitution», en M. Rosenfeld y A. SAjó (editores), The Oxford Handbook of Comparative Constitutional Law, Oxford, Oxford University Press, pp. 874-888, en p. 876. 
TITLE: «The constitutional regulation of Political Parties in the European Union: a sui generis balance»

ABSTRACT: Balance in political parties regulation is met in contemporary democracies when the attribution of relevant political functions to political parties is counterweighed with mechanisms of control. The application of this principle to the regulation of political parties under the EU legal order reveals a sui generis balance: the functions of European political parties in European elections or in the legislative process within the European Parliament is significantly limited by the leading role played by member States and national party politics; besides, the mechanisms of control on European political parties, including those of militant democracy, must coexist with national laws governing the same subject, creating the typical problems of multilevel scenarios.

RESUMEN: El equilibrio en la regulación de los partidos politicos en las democracias contemporáneas se alcanza cuando la atribución a estos de funciones políticas relevantes se compensa con mecanismos de control. La aplicación de este principio a la regulación de los partidos políticos en el derecho de la UE pone de manifiesto una situación de equilibrio sui generis: las funciones de los partidos politicos europeos en las elecciones europeas o en el proceso legislativo del Parlamento Europeo está fuertemente limitada por el protagonismo de los Estados miembros y la política de partidos nacionales; por otro lado, los mecanismos de control establecidos sobre los partidos políticos europeos, incluidos los de democracia militante, deben coexistir con las normas nacionales al respecto, generando los problemas típicos de los escenarios multinivel.

KEY WORDS: European political parties, State of political parties, European Union.

Palabras clave: Partidos políticos europeos, Estado de partidos, Unión Europea.

FECHA DE RECEPCIÓN: 15.06.2016

FECHA DE ACEPTACIÓN: 27.07.2016 
